\title{
Ressignificando o processo de notificação compulsória na perspectiva da Educação Permanente em Saúde: Um relato de experiência
}

\author{
Resignifying the compulsory notification process from the perspective of Permanent Health \\ Education: An experience report \\ Replanteando el proceso de declaración obligatoria en la perspectiva Educación Permanente en \\ Salud: Un informe de experiencia
}

Recebido: 26/04/2021 | Revisado: 05/05/2021 | Aceito: 12/05/2021 | Publicado: 28/05/2021

\author{
Tatiana Feliciano \\ ORCID: https://orcid.org/0000-0001-8816-1965 \\ Universidade Federal Fluminense, Brasil \\ E-mail: tatianafeliciano@id.uff.br \\ Benedito Carlos Cordeiro \\ ORCID: https://orcid.org/0000-0001-6387-511X \\ Universidade Federal Fluminense, Brasil \\ E-mail: bcordeiro@id.uff.br
}

\begin{abstract}
Resumo
A Educação Permanente em Saúde (EPS) apresenta desafios e estratégias para a melhoria da qualidade do atendimento em saúde. Por meio dela, o conhecimento e as experiências serão um bem comum a todos, de acordo com as perspectivas da Política Nacional de Educação Permanente em Saúde (PNEPS). Em vista disso, é necessário dar um novo significado ao processo de notificação compulsória, a fim de auxiliar na promoção, prevenção e controle das doenças, agravos e eventos de saúde pública. Entende-se que EPS é a estratégia capaz de proporcionar ao profissional de saúde a significância e relevância que esta área do conhecimento necessita. Nesse contexto, este estudo tem como objetivo apresentar o relato de experiência sobre os desafios da EPS na construção do Produto Tecnológico Educacional em relação à notificação compulsória. Os resultados demonstraram que o Produto Tecnológico Educacional, em consonância com as metas da PNEPS, como a estratégia de ensino-aprendizagem através de videoaulas para a ressignificação do processo de notificações compulsórias aos profissionais de saúde, é um meio capaz de contribuir com a reconstrução do conhecimento, atitudes e práticas no contexto da Vigilância Epidemiológica. Apesar de inúmeras referências bibliográficas fazerem menção à relevância da EPS em relação à subnotificação e melhoria da qualidade dos dados das Fichas de Notificação Compulsória, não foram identificados estudos discutindo de forma prática e aplicável estratégias para superar este problema.
\end{abstract}

Palavras-chave: Vigilância epidemiológica; Educação continuada; Notificação compulsória; Ensino na saúde.

\begin{abstract}
The Permanent Education in Health policy (EPS) presents challenges and strategies for the improvement of health care quality. Through it, knowledge and experiences will be a common good to all, according to the perspective of the National Policy for Permanent Education in Health (PNEPS). In light of this, it is necessary to give a new meaning to the compulsory notification process, in order to contribute to the promotion, prevention and control of diseases, grievances and public health events. We believe that EPS is the strategy capable of providing health care professionals with the understanding of the significance and relevance that this field of knowledge needs. In this context, this study aims to present the experience report on the EPS challenges faced in the construction of the Educational Technological Product regarding compulsory notification. The results show that the Educational Technological Product, in line with the PNEPS goals, as a teaching-learning strategy through video lessons to achieve the reframing of the process of compulsory notifications by health care professionals, is an effective means of contributing to the reconstruction of the knowledge, attitudes and practices in the context of Epidemiological Surveillance. Although countless bibliographic references mention the relevance of EPS regarding underreporting and improving the data quality in Compulsory Notification Sheets, no studies were identified discussing strategies to overcome this problem in a practical and applicable way.
\end{abstract}

Keywords: Epidemiological surveillance; Continuing education; Compulsory notification; Health education.

\section{Resumen}

La Educación Permanente en Salud presenta retos y estrategias para la mejora de la calidad de las consultas médicas. A través de ella, el conocimiento y las experiencias serán un bien común a todos, segundo las perspectivas de la Política 
Nacional de Educación Permanente en Salud. En vista de eso, es necesario conferir un nuevo significado al proceso de declaración obligatoria, a fin de asistir la promoción, prevención y control de las enfermedades, agravios y eventos de salud pública. Se entiende que la Educación Permanente en Salud (EPS) es la estrategia para proporcionar al profesional de salud la significación y relevancia que esta área del conocimiento necesita. En ese contexto, el objetivo de este estudio es presentar el informe de la experiencia sobre los retos de la EPS en la construcción de lo Producto Tecnológico Educacional en relación a la declaración obligatoria. Los resultados demuestran que el Producto Tecnológico Educacional, en consonancia con las metas da de la Política Nacional de Educación Permanente en Salud, como la estrategia de encino y aprendizaje a través de videoaulas por el replanteamiento del proceso de declaraciones obligatorias a los profesionales de salud, es un medio para la reconstrucción del conocimiento, actitudes y prácticas en el contexto de la Vigilancia Epidemiológica. A pesar de la relevancia de la Educación Permanente en Salud en relación a la su notificación y mejora de la calidad de datos de los archivos de declaración obligatoria, no fueron identificados estudios discutiendo de manera práctica y aplicable estrategias para superar este problema.

Palabras clave: Vigilancia epidemiológica; Educación continua; Declaración obligatoria; Educación para la salud.

\section{Introdução}

\section{Perspectivas da Educação Permanente em Saúde}

A Educação Permanente em Saúde (EPS) possui desafios e estratégias para a melhoria da qualidade do atendimento em saúde. Neste contexto, Merhy (2013), com sua vasta reflexão e discussão sobre este tema, aborda a questão do coletivo para o coletivo. Os saberes e incertezas identificados no mundo do trabalho devem ser compartilhados, ao invés de represados. Desta forma, o conhecimento e as experiências serão um bem comum a todos, de acordo com as perspectivas da Política Nacional de Educação Permanente em Saúde (PNEPS).

Ao pesquisar sobre o aprimoramento da Rede e Promoção do Acesso aos Serviços de Saúde, Merhy e colaboradores (2016) abordam o pensar em EPS, como refletir constantemente sobre a prática, de modo a atuar para trilhar caminhos que colaborem com a "desterritorialização", ou seja, agir em função da desfragmentação do cuidado em saúde. Sendo assim, a EPS perpassa as esferas de apoio aos trabalhadores e gestores numa movimentação constante de seus pares para a indagação sobre o seu próprio espaço de trabalho.

"O que te incomoda no seu trabalho?". É através desta indagação que Feuerwerker e col. (2016) refletem sobre a EPS como um espaço que deve promover a discussão destes incômodos e transformá-los em motivadores para as mudanças nos processos relativos à educação na saúde. Pensar em Educação Permanente é entrar no campo dos conhecimentos, atitudes e práticas em saúde, considerando o conhecimento prévio do profissional, a fim de sensibilizá-lo para mudanças de suas atitudes e a partir daí rever seu processo de prática profissional e focar no cuidado integral aos usuários dos serviços de saúde e nas suas relações de trabalho.

Sá e col. (2018) concluem em seu estudo que, na perspectiva de reorganizar o processo de trabalho, a EPS é um importante disparador de provocações que remetem o profissional a refletir sobre a sua prática. Sob o ponto de vista da Vigilância Epidemiológica, este conceito se adequa ao processo de notificação compulsória de doenças, agravos e eventos de saúde pública; fato este que, em consonância com um dos objetivos da PNEPS, propõe a mudança da execução das práticas de trabalho e da respectiva sistematização do serviço Brasil (2009).

Na tentativa de recuperar conhecimentos oferecidos durante a formação profissional, Ceccim (2005) aborda a EPS como um desdobramento desta formação específica para o mundo do trabalho. Entretanto, este pensamento pode ser perfeitamente aplicado aos conhecimentos oriundos da prática de trabalho, promovendo uma aprendizagem significativa capaz de inquietar e promover mudanças, sejam elas pessoais, institucionais e, quem sabe, até políticas, gerando novos conhecimentos, atitudes e práticas de trabalho.

\section{O processo de notificação compulsória na perspectiva da EPS}

A construção da identidade epidemiológica de uma determinada localidade tem seu início na coleta dos dados; 
entretanto, quando esta construção é falha, gera a incompletude das notificações e, consequentemente, uma identidade equivocada da situação de saúde/doença e desenho epidemiológico distorcido de uma determinada região ou localidade (Abath et al., 2014).

Diante disso, é necessário oferecer estímulos de aprimoramento ao conhecimento humano, a fim de se elevar o empenho do profissional no desenvolvimento de suas atividades, que neste caso trata-se do processo de notificação de doenças, agravos e eventos de saúde pública. Desta maneira, a Vigilância Epidemiológica (VE), responsável pela organização destes dados e informações, desenha os padrões das ocorrências e fatores de saúde pública de uma determinada região o mais próximo possível da sua realidade (Guimarães et al., 2019).

A definição do Ministério da Saúde (MS) para EPS, como ação de promoção e aquisição de conhecimentos no ambiente de prática, é adequada e coerente para a proposta de ressignificação do processo de trabalho, bem como ao ato de notificar (Brasil, 2018). Sem dúvida, a PNEPS é capaz de possibilitar e cultivar o ensino-aprendizagem aos profissionais de saúde, mas apesar dos seus avanços e inserção nos espaços de discussão, ainda apresenta lacunas e um vasto campo de trabalho carente por essa política.

A proposta da PNEPS para a formação e desenvolvimento dos trabalhadores do setor para o SUS possui uma perspectiva coletiva e ampliada, pois ao mesmo tempo que oferece conhecimento aos profissionais, retorna com melhoria no atendimento e no serviço aos usuários. Em se tratando da proposta de dar um novo significado ao processo de notificação compulsória, ganhase em informação, conhecimento e investimentos mais direcionados à saúde (Brasil, 2004).

A comunicação em saúde é realizada através de diversas formas e modelos sugeridos pelo Sistema Único de Saúde (SUS); entretanto, a notificação compulsória mediante a suspeita ou confirmação de doença, agravo ou evento de saúde pública que compõe a Lista Nacional de Doenças de Notificação é considerada uma fonte de informação rica e completa quando preenchida em sua totalidade.

Para tanto, foi implementada em 1975 a Lei Federal nº 6.259 (Brasil, 1975), que dispõe sobre a obrigatoriedade da notificação das doenças pelos profissionais e usuários descritos. Com o passar do tempo, as transformações mundiais e tecnológicas, alguns pontos foram alterados e outros inclusos, mas sem alterar o objetivo principal desta lei.

Embora já seja reconhecida e estabelecida a relevância da notificação para a saúde pública, ainda estamos caminhando a passos curtos neste processo. $\mathrm{O}$ ato de notificar a suspeita ou confirmação de uma doença, agravo ou evento de saúde pública ainda é desconsiderado por profissionais de saúde, provavelmente por considerá-lo como trabalho burocrático, sem valor, acúmulo de serviço ou outra característica que o impeça de realizar a notificação.

$\mathrm{O}$ registro correto das informações propicia o entendimento de como os agravos/casos ocorrem, para intervir o mais precocemente possível. O tratamento inadequado, as dificuldades na busca ativa dos casos, a disseminação de doenças, a incontingência de surtos e outros problemas são identificados por meio de instrumentos de coleta de dados da vigilância em saúde como, por exemplo, a Ficha de Notificação.

Quando as fichas não são utilizadas corretamente ou são subutilizadas, por ignorar variáveis importantes e registrar informações equivocadas por falta de conhecimento técnico, impedem a atuação efetiva no controle e prevenção de doenças, agravos e eventos de saúde pública (Ferreira et al., 2018; Souza et al., 2017).

Em vista disso, é necessário dar um novo significado ao processo de notificação compulsória a fim de auxiliar na promoção, prevenção e controle das doenças, agravos e eventos de saúde pública. Portanto, entende-se que EPS é a estratégia capaz de proporcionar ao profissional de saúde a significância e relevância que esta área do conhecimento necessita.

Nesse contexto, este estudo tem como objetivo apresentar a experiência sobre os desafios na construção do Produto Tecnológico Educacional em relação à notificação compulsória na perspectiva da EPS. 


\section{Metodologia}

A metodologia selecionada para se atingir o objetivo deste estudo é a abordagem qualitativa do tipo relato de experiência, pois, segundo Minayo (2001, p. 07), este tipo de pesquisa não é palpável, não podendo ser medida através de cálculos. A abordagem qualitativa se adequa a este estudo pelo fato de permitir a construção de um plano para se atingir o objetivo proposto e responder a questão que norteia este estudo.

Certamente, o relato de experiência é adequado para uma abordagem qualitativa, na medida em que ele se apresenta de forma criativa e leve; porém, se bem estruturado, garante a proposta de melhor compreensão do contexto, de acordo com Godoy (1995, p. 21).

O presente relato de experiência descreve o caminho percorrido para a definição da estratégia de EPS a fim de ressignificar o processo de notificação compulsória, que é o Produto Tecnológico Educacional proposto e a motivação para este tema surge através de uma inquietação pessoal e dos problemas oriundos da prática de trabalho, em consonância com os objetivos da EPS. Apesar de atuar por dez anos na assistência à saúde, nos seus três níveis de atenção, a inquietação retrata a frequente não notificação de doenças, agravos ou eventos de saúde pública durante a prática profissional, dentre outros fatores.

A partir de então, iniciou-se o processo de inserção e adaptação a funções nunca antes desempenhadas, o que gerou medo, incerteza e insegurança, mesmo diante da experiência dos profissionais, que foram realocados fora das suas funções habituais e especializações específicas.

A definição da estratégia de ensino-aprendizagem para a produção do Produto Tecnológico Educacional faz parte da dissertação de Mestrado intitulada: "Análise da qualidade dos dados das Fichas de Notificação Compulsória de Dengue e Chikungunya: estratégia de Educação Permanente em Saúde”, aprovada pelo Parecer Consubstanciado do Conselho de Ética e Pesquisa do Hospital Universitário Antônio Pedro/Faculdade de Medicina da Universidade Federal Fluminense - HUFM/UFF sob o número CAAE 29256319.5.0000.5243 e anexos relacionados como declaração de riscos e benefícios, assim como o Termo de Consentimento Livre e Esclarecido.

O Produto Tecnológico Educacional é uma das exigências dos Mestrados Profissionais, hoje representada pela Portaria n 389, de 23 de março de 2017, no âmbito da Fundação Coordenação de Aperfeiçoamento de Pessoal de Nível Superior - CAPES (ABMES, 2018).

Almejando atender aos critérios e exigências da Coordenação de Aperfeiçoamento de Pessoal de Nível Superior (CAPES) sobre a criação de Produto Tecnológico Educacional e visando devolver para o mundo do trabalho os conhecimentos adquiridos na comunidade acadêmica, a escolha da estratégia de ensino-aprendizagem se deu através de uma enquete, em consulta aos profissionais de saúde do município, respeitando as regras de segurança sanitárias atuais e que possa ser de fácil acesso e entendimento para todos os níveis de escolaridade.

\section{Resultados e Discussão}

\section{Definição da Estratégia de Ensino-aprendizagem}

Para se definir a estratégia de ensino-aprendizagem a ser utilizada, foi criada uma enquete no Google Forms denominada "Enquete - Educação Permanente em Saúde", em formato de múltipla escolha, enviada através de um aplicativo de mensagens instantâneas aos grupos de trabalho de profissionais de saúde do município a ser realizada a pesquisa no período de 03 a 10 de março de 2021.

Para iniciar a enquete, o voluntário foi informado sobre os objetivos e demais assuntos relevantes, culminando com o aceite ou não em participar da pesquisa. 
O participante foi informado e esclarecido sobre o caráter voluntário de sua participação, o número de registro da pesquisa no Conselho de Ética e Pesquisa, assim como os contatos da pesquisadora responsável. Foram obtidas 63 respostas de aceite para a participação da enquete.

Ainda nesta enquete, o voluntário deveria selecionar a estratégia de ensino-aprendizagem de sua preferência. Este questionamento teve o propósito de dar significado e relevância às formas de aprendizagem dos atores principais deste Produto Tecnológico Educacional.

A escolha teve como base a estratégia de ensino-aprendizagem que melhor se adequava ao perfil individual, como demonstrado na Figura 1. Vale ressaltar que cada voluntário poderia escolher mais de uma opção.

Figura 1 - Escolha da Estratégia de ensino-aprendizagem da Enquete.

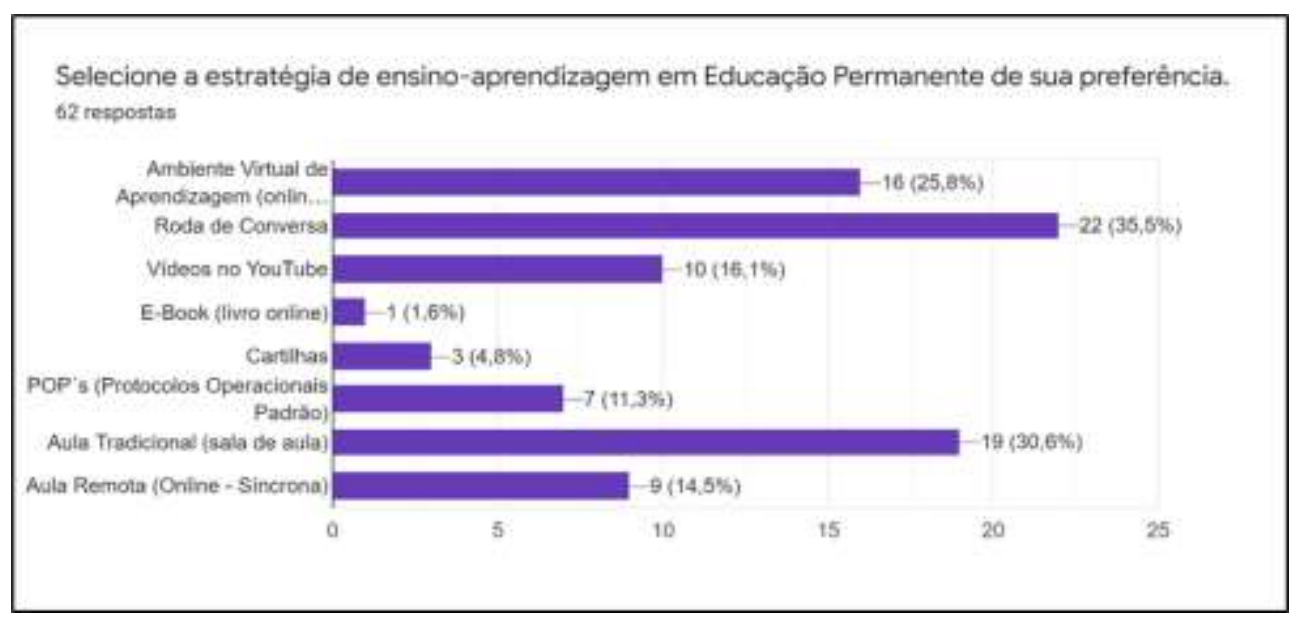

Fonte: Autores.

Após o encerramento da enquete, observou-se uma maior tendência aos métodos tradicionais de ensino-aprendizagem, apesar das orientações de evitar aglomerações e manter o distanciamento social devido a pandemia de COVID-19, pois alguns indivíduos relatam um melhor aprendizado desta forma.

Havia na enquete uma questão não obrigatória, que possibilitava ao participante justificar a sua resposta. Foram obtidas 27 respostas abordando diversos posicionamentos, porém o que chamou a atenção foi a incoerência entre a estratégia escolhida e as justificativas.

Desta forma, através dos relatos foi evidente a preocupação dos profissionais com a atual situação de saúde da população mundial devido à pandemia de COVID-19, conforme descrito nos relatos abaixo:

\footnotetext{
"As aulas presenciais são minha preferência, no entanto, neste momento de pandemia, opto pela aprendizagem on-line assincrona" $-R 1$;

"Atualmente, por conta da pandemia minha preferência é pelo ambiente virtual" - R7.
}

Apesar disso, devem-se ser levadas em consideração as formas de aprendizagem e a pouca habilidade através do ensino remoto.Talvez esse seja um dos motivos pelo qual as estratégias de ensino-aprendizagem tradicionais tenham sido a principal forma escolhida, conforme relatos:

\footnotetext{
"Tenho dificuldades nas aulas virtuais" - $R 2$;

"Acho válido aulas virtual e presencial, fica gravado em nossa memória fotográfica, gravamos e pra quem tem dislexia é uma opção que ajuda nos estudos" - R4.
} 
Vários fatores interferem nas estratégias de ensino-aprendizagem, dentre eles estão as questões financeiras para o deslocamento, violência urbana e a cultura tradicional de ensino. A proposta para este Produto Tecnológico Educacional se preocupa em atender as necessidades advindas do mundo do trabalho, propor soluções junto com os atores e retorná-las ao local de início com outra visão, outro pensamento e outras atitudes.

Desta forma, também foram levadas em consideração as expressões dos profissionais de saúde na enquete referentes à preocupação com a atual situação de saúde da população mundial provocada pela pandemia de COVID-19, como descrito abaixo:

“Com as possibilidades de hoje poder estarmos nos capacitando sem necessariamente termos que nos deslocar para realizarmos cursos, oficinas e outras capacitações, acho interessante uma modalidade que nos dá condições de economizar tempo e dinheiro, sem perder-se qualidade" - Rl8;

“Assim posso rever sempre que puder, e precisar" - R12;

"Posso acessar nas horas vagas" - R15.

\section{Proposta de Produto Tecnológico Educacional}

O Produto Tecnológico Educacional surgiu a partir de hipóteses confirmadas cientificamente e através do processo de trabalho da autora para este produto. Dentre as hipóteses estão: atualizações constantes nas definições dos casos, a ausência de assistência ao profissional que realiza notificações de violência, a imaturidade organizacional da gestão, a escassez de subsídios para facilitar a notificação, a falta de profissionais habilitados e treinados, a terceirização dos serviços de digitação e a escassez de tecnologias da informação nas unidades (Silva, et al., 2014; Carvalho, et al., 2011; Brustolin, et al., 2015 Garbin, et al., 2015).

Com o propósito de ofertar um produto em consonância com as metas do Programa Nacional de Educação Permanente em Saúde (PNEPS), a estratégia de ensino-aprendizagem escolhida foi o Ambiente Virtual de Aprendizagem, através da disponibilização das videoaulas em por meios digitais. Elegeu-se esta estratégia com o intuito de contribuir com a reconstrução do conhecimento, atitudes e práticas no contexto da Vigilância Epidemiológica.

Este Produto Tecnológico Educacional possui as seguintes características de acordo com os objetivos do mestrado profissional, conforme o Quadro 1:

Quadro 1 - Objetivos dos mestrados profissionais x características do Produto Tecnológico Educacional.

\begin{tabular}{|c|c|}
\hline Portaria Normativa $n^{0} 389-23 / 03 / 2017$ & Produto Tecnológico Educacional \\
\hline $\begin{array}{l}\text { I - Capacitar profissionais qualificados para o exercício da } \\
\text { prática profissional transformadora, atendendo as } \\
\text { demandas sociais, organizacionais ou profissionais do } \\
\text { mercado de trabalho; }\end{array}$ & $\begin{array}{l}\text { I - Capacitação dos profissionais de saúde em relação à } \\
\text { ressignificação do processo de notificação compulsória através de } \\
\text { estratégias de ensino-aprendizagem escolhidas pelos próprios atores } \\
\text { e para os atores; }\end{array}$ \\
\hline \multirow{2}{*}{$\begin{array}{l}\text { II - Transferir conhecimento para a sociedade, atendendo } \\
\text { as demandas específicas e de arranjos ao desenvolvimento } \\
\text { nacional, regional ou local; }\end{array}$} & $\begin{array}{l}\text { II - Estímulo a uma aprendizagem significativa dos profissionais de } \\
\text { saúde, a partir da demanda que surge do próprio mundo do trabalho, } \\
\text { a princípio numa perspectiva local, de modo a ressignificar os seus } \\
\text { conhecimentos, atitudes e práticas profissionais; }\end{array}$ \\
\hline & $\begin{array}{l}\text { III - Motivação e provocação ao tema Vigilância Epidemiológica } \\
\text { com vistas a intensificar a ação de notificação, meio este fornecedor } \\
\text { de informações imprescindíveis para as ações e estratégias em saúde } \\
\text { no país; }\end{array}$ \\
\hline $\begin{array}{l}\text { IV - Contribuir para agregar competitividade e aumentar a } \\
\text { produtividade em empresas, organizações públicas e } \\
\text { privadas. }\end{array}$ & $\begin{array}{l}\text { IV - Oferta de meios e técnicas possíveis para realizar notificações } \\
\text { compulsórias, segundo as demandas do SUS, em consonância com a } \\
\text { Portaria n. }{ }^{\circ} 204 \text { de } 17 / 02 / 2016 \text {. }\end{array}$ \\
\hline
\end{tabular}

Fontes: Autores, com base nas Portarias Normativas n. ${ }^{\circ}$ 389, de 23 de março de 2017, e n. ${ }^{\circ}$ 204, de 17 de fevereiro de 2016 (ABMES, 2017; Brasil, 2016). 


\section{Produto Tecnológico Educacional}

A estratégia para se realizar este produto foi definida de acordo com a situação atual de saúde mundial, já que, em virtude da pandemia de COVID-19, não é recomendado que ocorram encontros presenciais, evitando assim o aumento do contágio pelo vírus Sars-Cov2.

Apesar de $22 \%$ dos entrevistados terem selecionado a roda de conversa e 19\%, a aula tradicional como estratégia de ensino-aprendizagem através da enquete, é necessário respeitar as medidas sanitárias, pois o município em questão oscila entre a bandeira roxa e a vermelha em relação às medidas restritivas. Diante disso, elegeu-se a modalidade de videoaulas assíncronas disponibilizadas através de correio eletrônico (e-mail), grupos de trabalho pelo aplicativo de mensagens WhatsApp, e sediadas na plataforma de divulgação e interação através de vídeos em Canal no YouTube.

Desta maneira, foram criadas três videoaulas com os seguintes temas a serem discutidos, apresentados nos Quadros 2, 3 e 4:

Quadro 2 - Proposta videoaula I - Ressignificando o processo de notificação compulsória.

\begin{tabular}{|l|l|}
\hline Videoaula I - Ressignificando o processo de notificação compulsória I \\
\hline \multirow{4}{*}{ Título } & Aspectos conceituais, éticos e legais em notificação compulsória. \\
\hline \multirow{3}{*}{ Objetivos } & Definir o conceito de notificação compulsória; \\
\cline { 2 - 3 } & Descrever as aplicações práticas da notificação compulsória; \\
\cline { 2 - 3 } & Reafirmar as leis e portarias sobre notificação em saúde; \\
\cline { 2 - 3 } & Identificar na sua atividade as doenças, agravos e eventos de saúde pública. \\
\hline \multirow{3}{*}{ Tópicos } & I - Conceito de notificação compulsória; \\
\cline { 2 - 3 } & II - Aplicação prática das notificações; \\
\cline { 2 - 3 } & III - Aspectos éticos e legais da notificação compulsória; \\
\cline { 2 - 3 } & IV - Introdução às doenças, agravos e eventos de saúde pública de notificação. \\
\hline Aplicação & Videoaula com 30 minutos de duração e recursos de interação remota. \\
\hline
\end{tabular}

Fonte: Autores.

Quadro 3 - Proposta videoaula II - Ressignificando o processo de notificação compulsória.

\begin{tabular}{|l|l|}
\hline Videoaula II - Ressignificando o processo de notificação compulsória II \\
\hline \multirow{3}{*}{ Título } & Doenças, agravos e eventos de saúde pública de notificação compulsória. \\
\hline & Reconhecer as doenças, agravos e eventos de saúde pública; \\
\cline { 2 - 3 } & Investigar através dos critérios estabelecidos a inclusão/exclusão de suspeita; \\
\cline { 2 - 3 } & $\begin{array}{l}\text { Implementar a investigação dos critérios de inclusão/exclusão das doenças, agravos e eventos de saúde } \\
\text { pública. }\end{array}$ \\
\hline \multirow{3}{*}{ Tópicos } & Doenças, agravos e eventos de saúde pública de notificação compulsória; \\
\cline { 2 - 3 } & Principais Notificações Compulsórias em Itaboraí; \\
\cline { 2 - 3 } & Discussão de Casos Clínicos; \\
\cline { 2 - 3 } & Fichas de Notificação Compulsória. \\
\hline Aplicação & Videoaula com 30 minutos de duração e recursos de interação remota. \\
\hline
\end{tabular}

Fonte: Autores. 
Quadro 4 - Proposta videoaula III - Ressignificando o processo de notificação compulsória.

\begin{tabular}{|l|l|}
\hline Videoaula III - Ressignificando o processo de notificação compulsória III \\
\hline Título & Preenchendo a Ficha de Notificação Compulsória. \\
\hline \multirow{3}{*}{ Objetivos } & Compreender a importância da coleta de dados na anamnese e exame físico; \\
\cline { 2 - 3 } & Identificar os campos obrigatórios e essenciais da Ficha de Notificação; \\
\cline { 2 - 3 } & Analisar criticamente a Ficha de Notificação Compulsória. \\
\hline \multirow{3}{*}{ Tópicos } & Qualidade dos dados das Fichas de Notificação Compulsória; \\
\cline { 2 - 3 } & Ficha de Notificação Compulsória; \\
\cline { 2 - 3 } & Erros comuns no preenchimento das Fichas de Notificação Compulsória. \\
\hline Aplicação & Videoaula com 30 minutos de duração e recursos de interação remota. \\
\hline
\end{tabular}

Fonte: Autores.

A avaliação para a implementação deste Produto Tecnológico Educacional não se dará de forma imediata, ou seja, em curto prazo. Espera-se que o profissional de saúde possa ver-se como protagonista da vigilância em saúde nas suas atividades de trabalho e, assim, execute as suas atividades de trabalho com mais segurança e atenção em relação aos sinais e sintomas cabíveis para a suspeição ou confirmação de determinada doença, agravo ou evento de saúde pública. Também, através disso, reconstrua o significado de notificação compulsória, atendendo aos preceitos de gerar informações de saúde com qualidade e coerência, a fim de obter notificações com melhor eficiência nos dados e eficazes para desenhar o perfil epidemiológico do município.

Esse processo de ressignificação vem em consonância com os objetivos da proposta de Educação Permanente em Saúde, na tentativa promover uma aprendizagem significativa entre pares.

\section{Validação do Produto Tecnológico Educacional}

Com o propósito de dar validade ao produto, este foi adaptado do Instrumento de Validação de Conteúdo Educativo em Saúde (IVCES), a partir do trabalho de Leite e col. (2018), conforme o Quadro 5, com a finalidade de oferecer ao público-alvo um material condizente com o proposto. Desta forma, este instrumento também pode ser caracterizado como um pré-teste.

Devido às limitações de circulação e restrições sanitárias no período de construção deste produto e do instrumento de validação, a sua avaliação ocorreu de forma remota. As videoaulas e o IVCES foram enviados por e-mail, seguidos por um link que encaminhava os destinatários para o Google Forms. O instrumento foi adaptado em formato de formulário para se obter as respostas, sugestões e recomendações dos validadores do produto.

Os validadores deste produto foram selecionados segundo o envolvimento com a área de aderência referente ao tema proposto para a dissertação do Mestrado Profissional. Preservando as suas identidades, segue sua qualificação profissional atual. São eles dois professores do Mestrado, a coordenadora do Núcleo de Educação Permanente em Saúde, três enfermeiras do Núcleo de Vigilância Epidemiológica, três enfermeiros do Núcleo de Epidemiologia Hospitalar e dois coordenadores de Programa de Saúde da Atenção Básica. 
Quadro 5 - Instrumento de Validação de Conteúdo Educativo em Saúde.

\begin{tabular}{|c|c|c|c|c|}
\hline Itens avaliados & Descrição do item avaliado & $\mathbf{0}$ & 1 & 2 \\
\hline \multirow{5}{*}{$\begin{array}{l}\text { Objetivos: } \\
\text { propósitos, } \\
\text { metas } \\
\text { ou finalidades }\end{array}$} & 1. Contempla tema proposto & & & \\
\hline & 2. Adequado ao processo de ensino-aprendizagem & & & \\
\hline & 3. Esclarece dúvidas sobre o tema abordado & & & \\
\hline & 4. Proporciona reflexão sobre o tema & & & \\
\hline & 5. Incentiva a mudança de comportamento & & & \\
\hline \multirow{10}{*}{$\begin{array}{l}\text { Estrutura/Apresentação: } \\
\text { organização, } \\
\text { estrutura, } \\
\text { estratégia } \\
\text { e coerência }\end{array}$} & 6. Linguagem adequada ao público-alvo & & & \\
\hline & 7. Linguagem apropriada ao material educativo & & & \\
\hline & 8. Linguagem interativa & & & \\
\hline & 9. Informações corretas & & & \\
\hline & 10. Informações objetivas & & & \\
\hline & 11. Informações esclarecedoras & & & \\
\hline & 12. Informações necessárias & & & \\
\hline & 13. Sequência lógica das ideias & & & \\
\hline & 14. Tema atual & & & \\
\hline & 15. Tamanho do texto adequado & & & \\
\hline \multirow{3}{*}{$\begin{array}{l}\text { Relevância: significância, } \\
\text { impacto, motivação e interesse }\end{array}$} & 16. Estimula o aprendizado & & & \\
\hline & 17. Contribui para o conhecimento na área & & & \\
\hline & 18. Desperta interesse pelo tema & & & \\
\hline
\end{tabular}

Nota: Valoração dos itens: 0 discordo; 1 concordo parcialmente; 2 concordo totalmente. Fonte: Leite et al., 2018 [adaptado].

\section{Considerações Finais}

Este relato de experiência colaborou para a construção, organização e planejamento das atividades exigidas no Mestrado Profissional em Ensino na Saúde, além de promover o melhor entendimento sobre o tema em questão e permitir o aprofundamento no levantamento bibliográfico para a construção de um arcabouço teórico-ético-científico em relação à Educação Permanente em Saúde, na área da Vigilância Epidemiológica.

Apesar de inúmeras referências bibliográficas fazerem menção à relevância da Educação Permanente em Saúde em relação à subnotificação e melhoria da qualidade dos dados das fichas de notificação compulsória, não foram identificados estudos discutindo de forma prática e aplicável estratégias para superar este problema.

Recomendam-se mais estudos aprofundados sobre este assunto pouco explorado, porém de grande relevância para a tomada de decisões de gestores da saúde no enfrentamento de surtos, endemias, epidemias e pandemias.

\section{Referências}

Abath, M. B. et al. (2014). Avaliação da completitude, da consistência e da duplicidade de registros de violência do Sinan em Recife, Pernambuco, 2009-2012. Epidemiologia e Serviços de Saúde, 23(1),131-142.

Associação Brasileira de Mantenedoras do Ensino Superior (ABMES). (2017). Portaria MEC $n^{\circ} 389$, de 23 de março de 2017 . Dispõe sobre o mestrado e doutorado profissional no âmbito da pós-graduação stricto sensu.

Brasil. Ministério da Saúde. (1975). Lei n. ${ }^{\circ}$ 6.259, de 30 de outubro de 1975. Dispõe sobre as organizações de Vigilância Epidemiológica, sobre o Programa Nacional de Imunizações, estabelece normas relativas à notificação compulsória de doenças, e dá outras providências. Diário Oficial da União. Poder Executivo, Brasília, DF. 
Brasil. Ministério da Saúde (2009). Política Nacional de Educação Permanente em Saúde. Ministério da Saúde.

Brasil. Ministério da Saúde. (2016). Portaria n. ${ }^{\circ}$ 204, de 17 de fevereiro de 2016. Define a Lista Nacional de Notificação Compulsória de doenças, agravos e eventos de saúde pública nos serviços de saúde públicos e privados em todo território nacional.

Brustolin, P. X. \& Agusti, L. C. (2015). Conhecimento dos profissionais de saúde sobre os sistemas de informações em saúde e a sua importância. Universidade do Oeste de Santa Catarina - UNOESC. Trabalho de Conclusão do Curso de Pós Graduação em Saúde Coletiva. http://www.uniedu.sed.sc.gov.br/wpcontent/uploads/2016/09/unoesc-Lesiane-Cristina-Agusti.pdf

Carvalho, C. N., Dourado, I. \& Bierrenbach, A. L. (2011). Subnotificação da comorbidade tuberculose e AIDS: uma aplicação do método de linkage. Revista de Saúde Pública, 45(3), 548-555. http://dx.doi.org/10.1590/S0034-89102011005000021.5

Ceccim, R. B. (2005). Educação Permanente em Saúde: desafio ambicioso e necessário. Interface - Comunicação, Saúde, Educação, 9(16), 161-168.

Ferreira, L. P. et al. (2018). Distúrbio de voz relacionado ao trabalho: proposta de uma ficha de notificação individual. Distúrb. Comum., 30(1), 170-178.

Feuerwerker, L. C. M. et al (Orgs.). (2016). Avaliação compartilhada do cuidado em saúde: surpreendendo o instituído nas redes. Políticas e cuidado em saúde. Rio de Janeiro: Hexis.

Garbin, C. A. S., Dias, I. A., Rovida, T. A. S. \& Garbin, A. J. Í. (2015). Desafios do profissional de saúde na notificação da violência: obrigatoriedade, efetivação e encaminhamento. Ciência \& Saúde Coletiva, 20(6), 1879-1890. https://dx.doi.org/10.1590/1413-81232015206.13442014

Godoy, A. S. (1995). Pesquisa qualitativa - tipos fundamentais. Revista de Administração de Empresas, 35(3), 20-29.

Guimaraes, M. S. O., Andrade, J. X., Araújo, T. M. E., Oliveira, A. D. S. \& Mascarenhas, M. D. M. (2019). Avaliação da implantação dos Núcleos Hospitalares de Epidemiologia. Revista de Enfermagem UFPE online, 13(4), 1097-105.

Leite, S. S., Áfio, A.C. E., Carvalho, L. V., Silva, J. M., Almeida, P. C. \& Pagliuca, L. M. F. (2018) Construction and validation of an Educational Content Validation Instrument in Health. Revista Brasileira de Enfermagem, 71(4), 1635-41.

Minayo, M. C. S. (Org.). (2001). Pesquisa Social. Teoria, método e criatividade. Vozes.

Merhy, E. E. (2013). Micropolítica, instituições e governos dos vivos: plataforma de interinventividade a partir da Linha de Pesquisa Micropolítica do Trabalho e Cuidado em Saúde. Saúde Micropolítica - Blogspot. http://saudemicropolitica.blogspot.com/.

Merhy, E. E. et al (Orgs.). (2016). Avaliação compartilhada do cuidado em saúde: surpreendendo o instituído nas redes. Políticas e cuidados em saúde. Hexis.

Ministério da Saúde (BR). (2004). Portaria. MS no 198, de 13 de fevereiro de 2004. Institui a Política Nacional de Educação Permanente em Saúde como estratégia do Sistema Único de Saúde para a formação e o desenvolvimento de trabalhadores para o setor dá outras providências. Diário Oficial da União. Poder Executivo.

Ministério da Saúde (BR). (2006). Dengue. Instruções para o preenchimento. Ficha de Investigação - Sinan NET. Ministério da Saúde.

Ministério da Saúde (BR). (2018). Política Nacional de Educação Permanente em Saúde: o que se tem produzido para o seu fortalecimento? Ministério da Saúde.

Sá, A. C. M. G. N. et al. (2018). Contribuições da Educação Permanente para qualificação da assistência de enfermagem em um hospital público. Revista Brasileira de Ciências da Saúde, 22(1), 87-94.

Silva, G. A. \& Oliveira, C. M. G. (2014). O registro das doenças de notificação compulsória: a participação dos profissionais de saúde e da comunidade. Revista de Epidemiologia e Controle de Infecção, 4(3), 215-220. https://doi.org/10.17058/reci.v4i3.4578

Souza, L. F. M. et al. (2017). Análise dos casos de notificação de sífilis congênita em um hospital de referência de Niterói, 2008-2015. DST - J. bras. Doenças Sex Transm, 29(1), 17-21. 